\title{
Hormigas (Hymenoptera: Formicidae) de Aguascalientes
}

\author{
Ants (Hymenoptera: Formicidae) of Aguascalientes
}

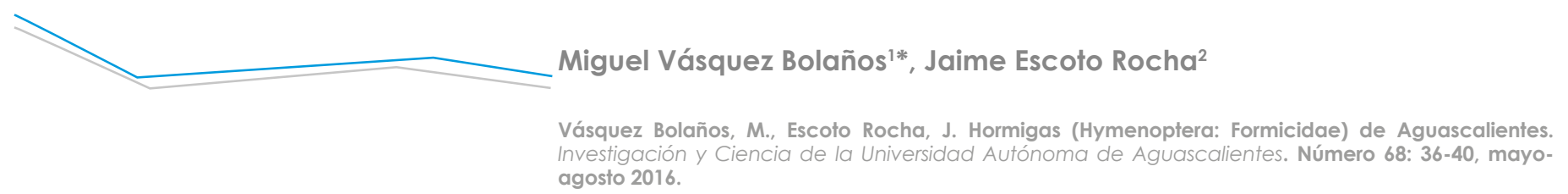

\section{RESUMEN}

Se elaboró una lista de especies de hormigas con base en los ejemplares depositados en las colecciones entomológicas de la Universidad Autónoma de Aguascalientes y de la Universidad de Guadalajara, así como con información obtenida en publicaciones especializadas sobre la familia Formicidae para el estado de Aguascalientes. Se conocen 32 especies de hormigas para el mismo: Atta mexicana, Pogonomyrmex barbatus, $P$. rugosus y Myrmecocystus melliger son de las que se tiene mayor número de ejemplares. El municipio de Aguascalientes es el que cuenta con el mayor número de especies registradas, 19; San José de Gracia con 11 y Calvillo con 10. En los municipios de Cosío, El Llano, Rincón de Romos y Tepezalá no se tienen especies de hormigas registradas. Se requiere mayor esfuerzo enfocado al estudio de hormigas en Aguascalientes.

Palabras clave: Formicidae, riqueza, colección, Aguascalientes.

Keywords: Formicidae, richness, collection, Aguascalientes.

\section{Recibido: 15 de abril de 2015, aceptado: 14 de diciembre de 2015}

Departamento de Botánica y Zoología, Centro Universitario de Ciencias Biológicas y Agropecuarias, Universidad de Guadalajara.

2 Departamento de Biología, Centro de Ciencias Básicas, Universidad Autónoma de Aguascalientes.

* Autor para correspondencia: mvb14145@hotmail.com

\section{ABSTRACT}

A list of ant species was assembled based on specimens from the entomological collections of the Universidad Autonoma de Aguascalientes and the Universidad de Guadalajara, as well as from information of Formicidae of Aguascalientes. The species richness of ants found for Aguascalientes is 32 . Atta mexicana, Pogonomyrmex barbatus, P. rugosus and Myrmecocystus melliger are the species with the largest number of specimens. The municipality of Aguascalientes has the largest richness, with 19 species; whereas San Jose de Gracia with 11 and Calvillo with 10 species. There are no records of ants in the municipalities of Cosio, El Llano, Rincon de Romos and Tepezala. More effort focused on the studies of ants in Aguascalientes is required.

\section{INTRODUCCIÓN}

Las hormigas son un grupo de insectos sociales, diverso y abundante; que junto con las abejas y avispas integran el orden Hymenoptera (Hölldobler y Wilson, 1990). Todas las especies de estos insectos son eusociales y se agrupan en la familia Formicidae (Bolton, 2003), las colonias están formadas por castas diferenciadas que cumplen una tarea en particular: las reinas y los machos cumplen la función reproductiva, generalmente presentan alas; las obreras (hembras estériles) se encargan del resto de las tareas dentro y fuera de la colonia, son ápteras (Hölldobler y Wilson, 1990). Se localizan en casi todos los ambientes terres- 
tres, están presentes en las siete regiones biogeográficas, los polos y picos de altas montañas no han sido colonizados por hormigas (Fernández, 2003; Fernández y Sendoya, 2004). A nivel mundial se conocen casi 13,000 especies distribuidas en 290 géneros y 20 subfamilias (Agosti y Johnson, 2005). La región neotropical es la más diversa con aproximadamente 3,100 especies, 95 géneros y 12 subfamilias (Agosti y Johnson, 2005; Bolton et al., 2006; Ward, 2010). Para México se conocen 927 especies de hormigas, pertenecientes a 93 géneros y 11 subfamilias; el sureste y el norte del país son las zonas en la que se conoce la mayor riqueza (Vásquez Bolaños, 2015). Para el estado de Aguascalientes, de acuerdo con la literatura, se tienen registradas 17 especies, pertenecientes a 11 géneros y cuatro subfamilias (Cañedo Ortiz, 1988; Escoto Rocha et al., 2001; Vásquez Bolaños, 2015). A pesar de su importancia son un grupo poco estudiado en México, no se tienen especialistas ni colecciones suficientes para conocer la riqueza y distribución del grupo en el país (Vásquez Bolaños, 2011). El objetivo de este trabajo es conocer la riqueza de hormigas en el estado de Aguascalientes con base en los ejemplares depositados en colecciones y registros en la literatura.

\section{MATERIALES Y MÉTODOS}

El estado de Aguascalientes tiene una superficie de $5,680.33 \mathrm{~km}^{2}$, se localiza en el centro del país, en las coordenadas extremas $22^{\circ} 27^{\prime}$ y $21^{\circ} 28^{\prime}$ latitud norte y $101^{\circ} 53^{\prime}$ y $102^{\circ} 52^{\prime}$ longitud oeste. Limita al norte, este y oeste con el estado de Zacatecas, al sur y al este con el estado de Jalisco; tiene 11 municipios: Aguascalientes, Asientos, Calvillo, Cosío, El Llano, Jesús María, Pabellón de Arteaga, Rincón de Romos, San Francisco de los Romo, San José de Gracia y Tepezalá. Se encuentra a 1,540 m.s.n.m., en la parte baja del valle del río Calvillo, hasta los 3,050 m.s.n.m. en la parte alta de la Sierra Fría (Esparza Juárez, 2008; INEGI, 2008a; INEGI, 2008b).

Se revisaron dos colecciones entomológicas: la Zoológica del Centro de Ciencias Básicas de la Universidad Autónoma de Aguascalientes (UAA) y la Entomológica del Centro de Estudios en Zoología de la Universidad de Guadalajara (CZUG). Los ejemplares fueron determinados o corroborados para elaborar la lista. Se revisó literatura especializada sobre hormigas en las bibliotecas de estas y otras universidades que hiciera referencia al estado de Aguascalientes: libros, artículos en revistas científicas, tesis, resúmenes de congresos.

\section{RESULTADOS}

Se encontraron 32 especies, pertenecientes a 19 géneros y seis subfamilias para el estado de Aguascalientes (Tabla 1). El municipio en el que se ha colectado el mayor número de especies es Aguascalientes, con 19; seguido de los municipios de San José de Gracia con 11 y Calvillo con 10 especies; Jesús María con cinco; San Francisco de los Romo, Pabellón de Arteaga y Asientos con una cada uno; para los municipios de Cosío, El Llano, Rincón de Romos y Tepezalá no se tienen especies de hormigas registradas. Las especies Camponotus sericeiventris, C. trepidulus, Nylanderia bruesii, Pheidole calens, P. laevivertex y Pogonomyrmex tenuispinus no tienen localidad precisa (Tabla 2).

De las especies que se encuentran depositadas en las colecciones, A. mexicana, $P$. barbatus, $P$. rugosus y $M$. melliger son las que están mejor representadas, por el número de ejemplares. Los géneros más diversos son: Camponotus, Pogonomyrmex, Pheidole y Solenopsis con tres cada uno; Dorymyrmex, Forelius, Monomorium, Neivamyrmex y Pseudomyrmex con dos cada uno, el resto de los géneros (10) están representados por una especie cada uno. La subfamilia más diversa es Myrmicinae con 15 especies y ocho géneros, Formicinae con seis y cuatro, Dolichoderinae con cinco y tres, Dorylinae con tres y dos, Pseudomyrmecinae con dos y uno, respectivamente, y Ponerinae con una especie y un género también.

\section{DISCUSIÓN}

De las 32 especies hasta ahora conocidas para el estado de Aguascalientes, se tienen dos introducidas: Paratrechina longicornis y Monomorium pharaonis, originarias de Asia y África, respectivamente. Estas se

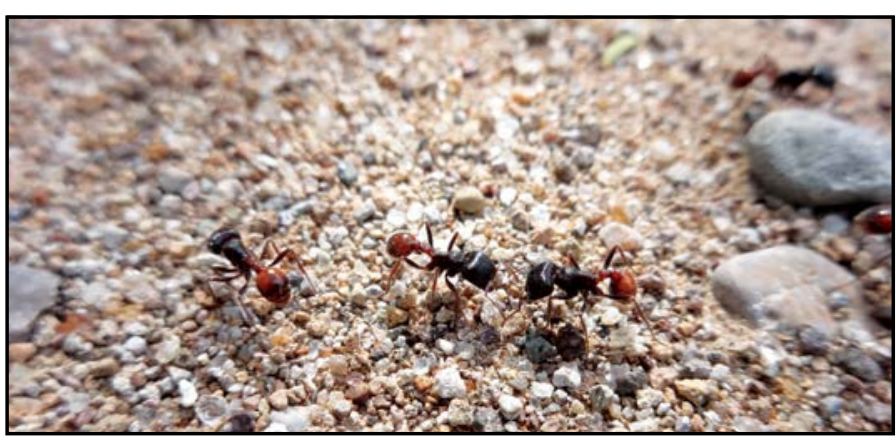

Figura 1. Obreras de Pogonomyrmex rugosus. Fotografía tomada por Miguel Vásquez Bolaños. 
Tabla 1. Lista de especies de hormigas para el estado de Aguascalientes

Formicidae Latreille, 1809

Dolichoderinae Forel, 1878

Leptomyrmecini Emery, 1913

Dorymyrmex Mayr, 1866

Dorymyrmex insanus (Buckley, 1866)

Forelius Emery, 1888

Dorymyrmex pyramicus (Roger, 1863)

Forelius mccooki (McCook, 1880)

Tapinomini Emery, 1913

Forelius pruinosus (Roger, 1863)

Liometopum Mayr, 1861

Liometopum apiculatum Mayr, 1870

Formicinae Latreille, 1809

Campinotini Forel, 1878

Camponotus Mayr, 186

Camponotus atriceps (Smith, 1858)

Camponotus sericeiventris (Guérin-Méneville, 1838)

Lasini Ashmead, 1905

Camponotus trepidulus Creigthon, 1965

Myrmecocystus Wesmael, 1838

Myrmecocystus melliger Forel, 1886

Plagiolepidini Forel, 1866

Nylanderia Emery, 1906

Nylanderia bruesii (Wheeler, 1903)

Paratrechina Motschoulsky, 1863

Paratrechina longicornis (Latreille, 1802)

Pseudomyrmecinae Smith, 1952

Pseudomyrmecini Smith, 1952

Peudomyrmex Lund, 1831

Pseudomyremx gracillis (Fabricius, 1804)

Pseudomyremx pallidus (Smith, 1855)

Dorylinae Leach, 1815

Dorylini Leach, 1815

Labidus Jurine, 1807

Labius coecus (Latreille, 1802)
Neivamyrmex Borgmeier, 1940

Neivamyrmex cornutus Watkins, 1975

Neivamyrmex texanus Watkins, 1972

Ponerinae Lepeletier, 1835

Ponerini Lepeletier, 1835

Odontomachus Latreille, 1804

Odontomachus clarus Roger, 1861

Myrmicinae Lepeletier, 1835

Attini Smith, 1858

Atta Fabricius, 1818

Atta mexicana (Smith, 1858)

Pheidole Westwood, 1839

Pheidole calens Forel, 1901

Pheidole hirtula Forel, 1899

Pheidole laevivertex Forel, 1901

Crematogastrini Forel, 1893

Crematogaster Lund, 1831

Crematogaster lineolata (Say, 1836)

Tetramorium Mayr, 1855

Tetramorium spinosum (Pergande, 1896)

Pogonomyrmecini Ward, Brady, Fisher \& Schultz, 2015

Pogonomyrmex Mayr, 1868

Pogonomyrmex barbatus (Smith, 1858)

Pogonomyrmex rugosus Emery, 1895

Pogonomyrmex tenuispinus Forel, 1914

Solenopsidini Forel, 1893

Monomorium Mayr, 1855

Monomorium minimum (Buckley, 1867)

Monomorium pharaonis (Linnaeus, 1758)

Solenopsis Westwood, 1840

Solenopsis aurea Wheeler, 1906

Solenopsis geminata (Frabicius, 1804)

Solenopsis xyloni McCook, 1880

Stenammini Ashmead, 1905

Aphaenogaster Mayr, 1853

Aphaenogaster cockerelli André, 1893 encuentran ampliamente distribuidas en el planeta y se consideran plagas urbanas (Bajomi et al., 2005; Wetterer, 2008), las mismas figuran en la zona urbana del municipio de Aguascalientes.

El estado de Aguascalientes se encuentra entre los siete estados del país con el menor número de especies de hormigas conocidas, con más especies que el Distrito Federal, Guanajuato, Estado de México, Querétaro, Tlaxcala y Zacatecas (Vásquez Bolaños, 2011). La entidad comparte el tipo de vegetación y clima con los estados de Jalisco y Zacatecas, por la proximidad es posible que algunas especies se compartan; por lo que el número de las mismas se incrementará de manera considerable cuando se realicen más estudios enfocados a conocer la riqueza de Formicidae en el estado.
La riqueza de las diferentes subfamilias coincide con lo que se conoce a nivel regional, nacional y mundial, donde Myrmicinae está en primer lugar, seguida de Formicinae, Dolichoderinae, Dorylinae y Pseudomyrmecinae (Bolton, 2003; Vásquez Bolaños, 2011).

Al contrastar la extensión territorial y la complejidad de los ecosistemas que se presentan en el estado (por su ubicación entre las regiones neártica y neotropical) con el reducido número de especies reportadas y los pocos ejemplares depositados en colecciones entomológicas, queda claro que la familia Formicidae no ha recibido la debida atención por parte de los investigadores; es mucho lo que se encuentra pendiente en cuanto a las hormigas del estado de Aguascalientes y de México. 


\section{IIVESEIGACIÓn

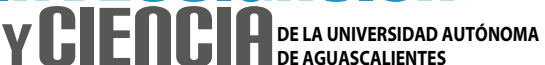

Tabla 2. Lista de especies de hormigas por municipio de Aguascalientes

\begin{tabular}{|c|c|c|c|c|c|c|c|c|}
\hline \multirow[b]{2}{*}{ Subfamilia } & \multirow[b]{2}{*}{ Especie } & \multicolumn{7}{|c|}{ Municipio } \\
\hline & & AGS & ASI & CAL & $J M$ & PA & SFR & SJG \\
\hline \multirow[t]{5}{*}{ Dolichoderinae } & Dorymyrmex insanus & $x$ & & & & & & $x$ \\
\hline & Dorymyrmex pyramicus & & & $x$ & & & & \\
\hline & Forelius mccooki & $x$ & & & & & & \\
\hline & Forelius pruinosus & $x$ & & & & & & \\
\hline & Liometopum apiculatum & & & & & & & $x$ \\
\hline \multirow[t]{6}{*}{ Formicinae } & Camponotus atriceps & $x$ & & $x$ & $x$ & & & $x$ \\
\hline & Camponotus sericeiventris & & & & & & & \\
\hline & Camponotus trepidulus & & & & & & & \\
\hline & Myrmecocystus melliger & $x$ & & $x$ & $x$ & & & $x$ \\
\hline & Nylanderia bruesii & & & & & & & \\
\hline & Paratrechina longicornis & $x$ & & & & & & \\
\hline \multirow[t]{3}{*}{ Dorylinae } & Labius coecus & & & $x$ & & & & \\
\hline & Neivamyrmex cornutus & & & $x$ & & & & \\
\hline & Neivamyrmex texanus & & & & & & & $x$ \\
\hline \multirow[t]{2}{*}{ Pseudomyrmecinae } & Pseudomyremx gracilis & $x$ & & $x$ & $x$ & & & \\
\hline & Pseudomyremx pallidus & $x$ & & & & & & \\
\hline Ponerinae & Odontomachus clarus & $x$ & & & & & & \\
\hline \multirow[t]{15}{*}{ Myrmicinae } & Aphaenogaster cockerelli & $x$ & & & & & & \\
\hline & Atta mexicana & $x$ & $x$ & $x$ & $x$ & $x$ & $x$ & $x$ \\
\hline & Crematogaster lineolata & & & & & & & $x$ \\
\hline & Monomorium minimum & $x$ & & & & & & \\
\hline & Monomorium pharaonis & $x$ & & & & & & \\
\hline & Pheidole calens & & & & & & & \\
\hline & Pheidole hirtula & $x$ & & $x$ & & & & \\
\hline & Pheidole laevivertex & & & & & & & \\
\hline & Pogonomyrmex barbatus & $x$ & & $x$ & & & & $x$ \\
\hline & Pogonomyrmex rugosus & $x$ & & $x$ & $x$ & & & $x$ \\
\hline & Pogonomyrmex tenuispinus & & & & & & & \\
\hline & Solenopsis aurea & $x$ & & & & & & \\
\hline & Solenopsis geminata & $x$ & & & & & & $x$ \\
\hline & Solenopsis xyloni & $x$ & & & & & & \\
\hline & Tetramorium spinosum & & & & & & & $x$ \\
\hline
\end{tabular}

AGS= Aguascalientes, ASI= Asientos, CAL= Calvillo, JM= Jesús María, PA= Pabellón de Arteaga, SFR= San Francisco de los Romo, SJG= San José de Gracia.

\section{CONCLUSIONES}

Con base en los ejemplares depositados en las colecciones Zoológica de la Universidad Autónoma de Aguascalientes (UAA), Entomológica de la Universidad de Guadalajara (CZUG) y en las publicaciones revisadas, llegan a 32 el número especies de hormigas para el estado de Aguascalientes. 
Con este estudio, el estado de Aguascalientes pasa del segundo lugar al séptimo a nivel nacional entre las entidades pobremente conocidas en cuanto a especies de hormigas se refiere.

Los estudios que hacen referencia a la familia Formicidae de Aguascalientes son escasos, solo hay una tesis (de licenciatura en la Universidad Autónoma de Aguascalientes) y dos artículos.

Las especies mejor representadas en las colecciones son: A. mexicana, P. barbatus, P. rugosus y M. melliger. Los municipios de Aguascalientes, Calvillo y San José de Gracia son los que tienen registrado el mayor número de especies.
Se tienen pocos ejemplares depositados en las colecciones científicas consultadas, lo que refleja el escaso trabajo realizado para el grupo en el estado de Aguascalientes y lo mucho que falta por hacer.

\section{Agradecimientos}

Los autores desean agradecer a las autoridades de las colecciones visitadas: Colección Zoológica del Centro de Ciencias Básicas de la Universidad Autónoma de Aguascalientes y la Colección Entomológica del Centro de Estudios en Zoología de la Universidad de Guadalajara, por las facilidades para revisar los ejemplares de hormigas depositados en dichas instituciones.

LITERATURA CITADA

- BAJOMI, D. et al. Elimination of pharaoh's ant, Monomorium pharaonis colonies with S-methoprene baits (Hymenoptera: Formicidae). En C. Y. Lee \& W. H. Robinson (Eds.), Proccedings of the Fifth International Conference on Urban Pests (65-72). Malaysia: P \& Y Design Network, 2005.

- BOLTON, B. Synopsis and classification of Formicidae. Memoirs of the American Entomological Institute, 71, 1-370, 2003.

- BOLTON, B. et al. Bolton's Catalogue of the Ants of the World: 1758-2005 [Disco compacto]. US: Harvard University Press, 2006.

- CAÑEDO ORTIZ, B. O. Distribución, biología e implicaciones ecológicas y económicas de la entomofauna de la familia Formicidae en el municipio de Aguascalientes. Tesis de Licenciatura en Biología. Aguascalientes, Aguascalientes, México: Universidad Autónoma de Aguascalientes, 1988.

- ESCOTO ROCHA, J. et al. Biodiversidad de himenópteros del estado de Aguascalientes. Investigación y Ciencia de la Universidad Autónoma de Aguascalientes, 9(24): 20-27, 2001.

- esparza juÁREZ, A. 1.1. Superficie. Capítulo 1. Descripción del medio físico. En H. A. Villegas \& A. Cruz Angón (Coords. y Comps.), La biodiversidad en Aguascalientes: estudio de estado (24). México: Comisión Nacional para el Conocimiento y Uso de la Biodiversidad (CONABIO)-Instituto del Medio Ambiente del Estado de Aguascalientes (IMAE)-Universidad Autónoma de Aguascalientes, 2008.

- FERNÁNDEZ, F. (Ed.). Introducción a las hormigas de la región Neotropical. Bogotá, Colombia: Instituto de Investigación de Recursos Biológicos Alexander von Humboldt, 2003.

- FERNÁNDEZ, F. y SENDOYA, S. List of neotropical ants (Hymenoptera: Formicidae). Biota Colombiana, 5(1): 3-93, 2004.

- HÖLLDOBLER, B. E. \& WILSON, O. The Ants. US: Harvard University Press, 1990.
INEGI (INSTITUTO NACIONAL DE ESTADÍSTICA Y GEOGRAFÍA). 1.2. Relieve. Capítulo 1. Descripción del medio físico. En H. A. Villegas \& A. Cruz Angón (Coords. y Comps.), La biodiversidad en Aguascalientes: estudio de estado (24-27). México: Comisión Nacional para el Conocimiento y Uso de la Biodiversidad (CONABIO)-Instituto del Medio Ambiente del Estado de Aguascalientes (IMAE)-Universidad Autónoma de Aguascalientes, 2008a.

- INEGI (INSTITUTO NACIONAL DE ESTADÍSTICA Y GEOGRAFÍA). 1.7. Vegetación primaria. Capítulo 1. Descripción del medio físico. En H. A. Villegas \& A. Cruz Angón (Coords. y Comps.), La biodiversidad en Aguascalientes: estudio de estado (4244). México: Comisión Nacional para el Conocimiento y Uso de la Biodiversidad (CONABIO)-Instituto del Medio Ambiente del Estado de Aguascalientes (IMAE)-Universidad Autónoma de Aguascalientes, 2008b.

- VÁSQUEZ BOLAÑOS, M. Lista de especies de hormigas (Hymenoptera: Formicidae) para México. Dugesiana, 18(1): 95-133, 2011.

- VÁSQUeZ BOLAÑOS, M. Taxonomía de Formiciade (Hymenoptera) para México. Métodos en Ecología y Sistemática, 10(1): 1-53, 2015.

- WARD, P. S. Taxonomy, Phylogenetics, and Evolution. En L. Lach, C. L. Parr \& K. L. Abbott (Eds.), Ant ecology (3-17). New York, US: Oxford University Press, 2010.

- WetTerer, J. K. Worldwide spread of the longhorn crazy ant, Paratrechina longicornis (Hymenoptera: Formicidae). Myrmecological News, 11, 137-149, 2008.

\section{De páginas electrónicas}

- $\quad$ AGOSTI, D. y JOHNSON, N. F. (Eds.). Antbase. World Wide Web electronic publication. Recuperada el 19 de febrero de 2013 , de antbase.org, version (05/2005). 